\title{
Role of Muscarinic Receptors in Hypoalgesia Induced by Crocin in Neuropathic Pain Rats
}

\author{
Hossein Ali Safakhah $\mathbb{D D}^{1,2}$ Abbas Ali Vafaei $\mathbb{D D}^{1,2}$ Azin Tavasoli $\mathbb{D}^{3},{ }^{3}$ Simin Jafari $\mathbb{D}^{3},{ }^{3}$ \\ and Ali Ghanbari (iD) \\ ${ }^{1}$ Research Center of Physiology, Semnan University of Medical Sciences, Semnan 3519899951, Iran \\ ${ }^{2}$ Department of Physiology, Faculty of Medicine, Semnan University of Medical Sciences, Semnan 3519899951, Iran \\ ${ }^{3}$ Student Research Committee, Semnan University of Medical Sciences, Semnan 3519899951, Iran \\ Correspondence should be addressed to Ali Ghanbari; ghanbari@semums.ac.ir
}

Received 28 February 2020; Revised 23 October 2020; Accepted 28 October 2020; Published 25 November 2020

Academic Editor: Mugimane Manjanatha

Copyright (c) 2020 Hossein Ali Safakhah et al. This is an open access article distributed under the Creative Commons Attribution License, which permits unrestricted use, distribution, and reproduction in any medium, provided the original work is properly cited.

\begin{abstract}
Objective. Crocin as an important constituent of saffron has antineuropathic pain properties; however, the exact mechanism of this effect is not known. The aim of this study was whether the hypoalgesic effect of crocin can be exerted through muscarinic receptors. Materials and Methods. In the present project, 36 male Wistar rats ( $200 \pm 20 \mathrm{~g})$ were used. Animals randomly divided into six groups (sham, neuropathy, neuropathy + crocin, neuropathy + atropine $0.5 \mathrm{mg} / \mathrm{kg}$, neuropathy + atropine $1 \mathrm{mg} / \mathrm{kg}$, and neuropathy + atropine $1 \mathrm{mg} / \mathrm{kg}+$ crocin). Neuropathy was induced by the chronic constriction injury (CCI) method on the sciatic nerve. Crocin and atropine was administered intraperitoneally during 14 days following the $14^{\text {th }}$ day after surgery. Pain response was detected every three days, two hours after each injection and 3 days following last injection. Mechanical allodynia and thermal hyperalgesia were detected using the Von Frey filaments and plantar test device, respectively. Results. CCI significantly reduced the paw withdrawal response to mechanical and thermal stimulus $(P<0.01$ and $P<0.05$, respectively). Crocin therapy significantly reduced mechanical allodynia and thermal hyperalgesia induced by CCI $(P<0.05)$. Atropine pretreatment significantly blocked the hypoalgesic effect of crocin $(P<0.05$ in mechanical allodynia and $P<0.01$ in thermal hyperalgesia). Fourteen days administration of atropine alone at a dose of $0.5 \mathrm{mg} / \mathrm{kg}$ but not $1 \mathrm{mg} / \mathrm{kg}$ significantly reduced CCI-induced mechanical allodynia at day 30 after surgery. Conclusion. Crocin significantly decreased CCI-induced neuropathic pain. The hypoalgesic effect of crocin was blocked by atropine pretreatment, which indicates an important role for muscarinic receptors in the effect of crocin.
\end{abstract}

\section{Introduction}

Neuropathic pain is one of the major challenges in medical centers throughout the world. Allodynia, hyperalgesia, and spontaneous pain are among the most current symptoms of neuropathic pain [1]. This long lasting problem occurs due to various peripheral and central mechanisms following somatosensory injuries or diseases [2, 3]. Several studies have revealed that the cholinergic system is involved in neuropathic pain. Ferrier and his colleagues reported that central muscarinic $\mathrm{M} 2$ receptors modulate neuropathic pain induced by traumatic nerve injury [4]. Furthermore, they showed that activation of muscarinic $M 2$ receptors of insular cortex reduce oxaliplatin-induced neuropathic pain in male rats [4]. It has been observed that intracerebroventricular injection of oxotremorine (nonselective muscarinic agonist) leads to the antinociceptive effect [5]. Donepezil, a cholinesterase inhibitor, through spinal muscarinic receptors reduces mechanical hyperalgesia in a rat model of spinal nerve ligation [6]. According to a prior report, it seems that donepezil-induced analgesia could be due to upregulation of muscarinic M2 receptors [7]. Recently, Mendes and his colleagues showed that LASSBio-873 (a pyrazolo[3, 4]pyrrolo[3, 4]pyridine derivative), as a new muscarinic agonist, relieves spinal nerve ligation- (SNL-) induced neuropathic pain in male rats [8]. It has been reported that atropine 
pretreatment suppresses acetyl-L-carnitine-induced analgesia in mice and rats [9]. Numerous chemical substances are presented for treatment and management of neuropathic pain; however, none of them have been effective in relieving pain completely [4]. In addition, some medications have several side effects that limit their use [10]; so, it is important to use treatments without side effects or minimal side effects that are effective.

Psychologically, the use of natural remedies is generally more acceptable than chemicals due to fewer side effects [11-13].

Herbal medicines have been used extensively throughout the world for years [14]. Saffron is a Mediterranean plant which contains several important bioactive constituents including crocin (color of saffron), safranal (odor of saffron), and picrocrocin (taste of saffron) [15-17]. Saffron (Crocus sativus L), a traditional medicine, has anti-inflammatory, antinociceptive, and antioxidative properties $[18,19]$. It has been shown that saffron has protective effects against hepatic disorders, asthma, depression, chronic pain, anxiety, stress, and memory deficits [20-25]. There are a few studies that show the interaction of saffron with neurotransmitter systems $[22,23]$. It has been reported that in an in vitro model, saffron extract inhibits neurotransmission of the glutamatergic system in the rat brain cortex [26]. On the other hand, it has been revealed that the saffron aqueous extract increases glutamate and dopamine contents of rat brain [27]. Studies on the memory revealed interaction of saffron with the cholinergic system [28]. Another study showed that saffron inhibits acetylcholinesterase (AChE) activity through interaction with binding sites of AChE [29]. Some of beneficial effects of saffron have been attributed to crocin [30]. It has been shown that crocin has the neuroprotective effect in animal models [31, 32]. Prior research revealed that behavioral impairments (schizophrenia-like behavior) induced by ketamine were inhibited by crocin [33]. Several animal studies have shown that crocin alleviates neuropathic pain [34-36]. Crocin amplified sciatic nerve function in crush injured rats through decreasing oxidative stress [37]. There is rare information about interaction of crocin with neurotransmitter systems in neuropathic pain.

The more the mechanisms are known, the more likely to find modern therapeutic strategies to management of neuropathic pain. Considering the interaction of saffron with the cholinergic system in neuropathic pain and analgesic effect of crocin, which is an important ingredient of saffron, the aim of the present study was whether the analgesic effect of crocin on the chronic constriction injury (CCI) neuropathic pain could occur through interaction with the cholinergic system.

\section{Materials and Methods}

2.1. Animals. In the present study, 36 Wistar male rats $(180 \pm 20 \mathrm{~g})$ were used. Animals were kept in a place with controlled temperature $(20 \pm 2 \mathrm{C}), 12$ hours light-dark cycles, and free access to food and water. Six rats were housed in each cage. All procedures were conducted according to
National Institutes of Health Guide for Care and Use of Laboratory Animals and were approved by Ethics Committee of Semnan University of Medical Sciences and Health Services (IR.SEMUMS.RES 92/332111).

2.2. Drug and Dosages. Ketamine $(80 \mathrm{mg} / \mathrm{kg})$, xylazine $(10 \mathrm{mg} / \mathrm{kg})$, crocin $(60 \mathrm{mg} / \mathrm{kg})$, and atropine sulfate $(0.5$ and $1 \mathrm{mg} / \mathrm{kg}$ ) were purchased from Sigma-Aldrich Co. Doses of drugs were selected based on previous studies [38, 39]. All drugs were used intraperitoneally. Both crocin and atropine were dissolved in physiologic saline.

2.3. Induction of Neuropathy. For inducing neuropathic pain, after anesthetizing (mixture of ketamine and xylazine) the rats, the upper thigh of the left leg was shaved, disinfected, and incised about $2 \mathrm{~cm}$ at the place of the left sciatic nerve. The exposed common sciatic nerve was separated from surrounding tissues and was ligated using four loose ligation (catgut chromic 4-0) $1 \mathrm{~mm}$ apart. At the end, the muscle and skin were closed using silk suture 4-0 [40]. Animals were kept in a cage individually until full consciousness was regained.

2.4. Experimental Protocol and Groups. Animals were randomly assigned into six groups (sham, CCI, CCI + crocin $60 \mathrm{mg} / \mathrm{kg}, \quad \mathrm{CCI}+$ atropine $\quad 0.5 \mathrm{mg} / \mathrm{kg}, \quad \mathrm{CCI}+$ atropine $1 \mathrm{mg} / \mathrm{kg}$, and CCI + crocin + atropine $1 \mathrm{mg} / \mathrm{kg}$ ) with 6 rats in each group. Two weeks after surgery, treatment with crocin was initiated and lasted for fourteen days during which for every 3 days, behavioral tests were performed (detection of the behavioral pain response was performed 2 hours after injection). To determine if therapeutic effects of treatments were continued, 3 days following the last injection of crocin (day 30), pain behaviors were examined. Atropine sulfate was injected 30 minutes before crocin during experiments (group received atropine + crocin). The experiments were carried out according to the timeline that is shown in Figure 1.

\subsection{Assessment of Pain Behavior of the Animals}

2.5.1. Mechanical Allodynia. After adapting to the environment, paw withdrawal threshold of animals was detected by Von Frey filaments [41]. Von Frey filaments, which are polyethylene hairs, are calibrated according to their diameters and each of which applies a certain amount of force (gram) to the surface. The hairs are used from low strength towards stronger one. Each filament is used three times with 10 seconds interval between each. If the animal responded to two consecutive stimulations, this number would be considered as the answer; otherwise, the experiment with the higher number would be continued. Sixty gram force was considered as the cutoff point.

2.5.2. Thermal Hyperalgesia. Paw withdrawal response to thermal stimulation was detected using the plantar test device [40]. After adaptation in the plantar test device, 


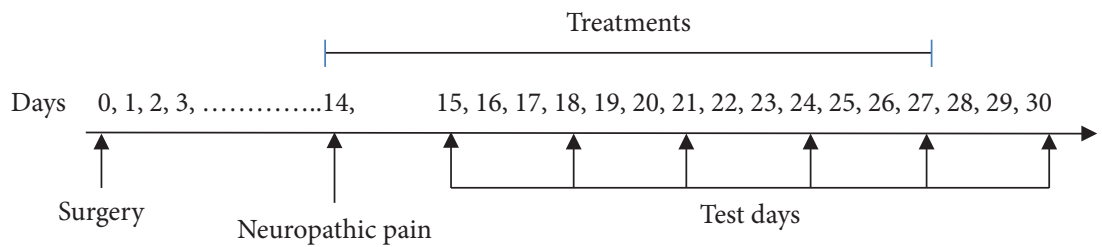

Figure 1: Timeline of experiments. Days $0,1,2,3, \ldots, 14,15,16,17,18,19,20,21,22,23,24,25,26,27,28,29$, and 30.

infrared beam source was focused on the injured paw's plantar surface, and irradiation of infrared was initiated with intensity equal to $60 \mathrm{~Hz}$. Latency of withdrawal response to thermal stimulation was recorded automatically. The test was performed 3 times with five minute intervals, and the average of these was considered as the paw withdrawal response. The cutoff point of test was adjusted at sixty seconds.

2.5.3. Statistical Analysis. Data analysis was performed by GraphPad prism version 8.0 software (GraphPad, San Diego, CA, USA). Regarding the normal distribution of data (using the Kolmogorov-Smirnov test), two-way analysis of variance (ANOVA) was used. Sidak's multiple comparisons test was used. All statistical tests were two-sided, and all data were expressed as mean \pm S.E.M of examined variable. $P<0.05$ was considered as the significant level.

\section{Results}

Our results showed that crocin alleviates CCI-induced neuropathic pain, and this effect was prevented using atropine pretreatment.

Chronic constriction injury of sciatic nerve significantly reduced withdrawal threshold response to mechanical and thermal stimulus at all days examined following surgery (Figures 2(a) and 2(b)). Crocin treatment significantly prevented CCI-induced mechanical allodynia $\left(F_{2,90}=72.57, \quad P<0.0001\right)$ and thermal hyperalgesia $\left(F_{2,90}=40.09, P<0.0001\right)$. After inducing neuropathic pain at day 14 after surgery, daily treatment with crocin began. Two weeks treatment with crocin significantly reduced mechanical allodynia $(P<0.001)$ and thermal hyperalgesia $(P<0.05)$. The analgesic effect of crocin continued for three days after stopping treatment (day 30 after surgery) (Figures 2(a) and 2(b)).

To determine the ineffective dose of atropine on the neuropathic pain, CCI neuropathic pain rats were subjected to atropine treatment from day 14 for fourteen days. Our results showed that atropine $(0.5 \mathrm{mg} / \mathrm{kg})$ as a muscarinic receptor antagonist could be effective on the neuropathic pain. Atropine with a dose of $0.5 \mathrm{mg} / \mathrm{kg}$ significantly $\left(F_{2,90}=7.8, \quad P=0.0007\right)$ decreased mechanical allodynia (Figure 3(a)) but not thermal hyperalgesia (Figure 3(b)) at day 30 after surgery (3 days after stopping treatment). However, atropine with a dose of $1 \mathrm{mg} / \mathrm{kg}$ did not change CCI-induced mechanical allodynia and thermal hyperalgesia during the time examined. Based on this result, a dose of $1 \mathrm{mg} / \mathrm{kg}$ atropine was selected for continuing to experiment.
According to our results, atropine with a dose of $1 \mathrm{mg} / \mathrm{kg}$ significantly prevented hypoalgesic effects of crocin. Animals received 14 days crocin therapy (i.p.) along with atropine (i.p.) pretreatment from day 14 after surgery. Our results showed that atropine significantly suppressed the improving effect of crocin on the mechanical allodynia $\left(F_{1,60}=7.266, \quad P=0.009\right) \quad$ and thermal hyperalgesia $\left(F_{1,60}=14.19, \quad P=0.0004\right)$ at day 27 after surgery (Figures 4(a) and 4(b), respectively). This inhibiting effect of atropine was continued on to $30^{\text {th }}$ day after surgery ( 3 days after which treatments have been stopped).

\section{Discussion}

In the present study, crocin improved neuropathic pain induced by CCI, and atropine prevented the crocin effect.

$\mathrm{CCI}$ as a model for inducing neuropathic pain like behavior leads to sign and symptoms similar to human's neuropathic pain [40]. In the present research, CCI led to clear mechanical allodynia and thermal hyperalgesia (Figure 2) that reached to a peak response at $14^{\text {th }}$ day after surgery. This is in agreement with our previous studies that 2 weeks after surgery mechanical allodynia and thermal hyperalgesia reached to the maximum level [42].

In this study, i.p. administration of crocin during 14 consecutive days following neuropathic pain clearly showed hypoalgesia (increased paw withdrawal threshold in response to mechanical and thermal stimulation).

In agreement with present results, it has already been reported that saffron and crocin (an active constituent of saffron) significantly reduce tactile allodynia and thermal hyperalgesia in neuropathic pain rats [36]. Furthermore, previously, we showed that saffron plays a significant role in reducing neuropathic pain [43].

There is no well-known mechanism presented for the hypoalgesic effect of crocin yet, but there are several mechanisms presented for neuropathic pain so far that have a prominent role [44-48]. It has been approved that the central cholinergic system plays an important role in pain processing so that activation of central cholinoceptors lead to antinociception $[45,49-51]$.

Since inhibition of the muscarinic cholinergic system could be effective on neuropathic pain, in the present study, it was necessary to detect the ineffective dose of atropine on the neuropathic pain. We examined the effect of atropine on the neuropathic pain with doses of $0.5 \mathrm{mg} / \mathrm{kg}$ and $1 \mathrm{mg} / \mathrm{kg}$ and observed that mechanical allodynia and thermal hyperalgesia at day 30 following CCI (the time that treatments had been finished three days ago) significantly reduced with a dose of $0.5 \mathrm{mg} / \mathrm{kg}$ but not with a dose of $1 \mathrm{mg} /$ 


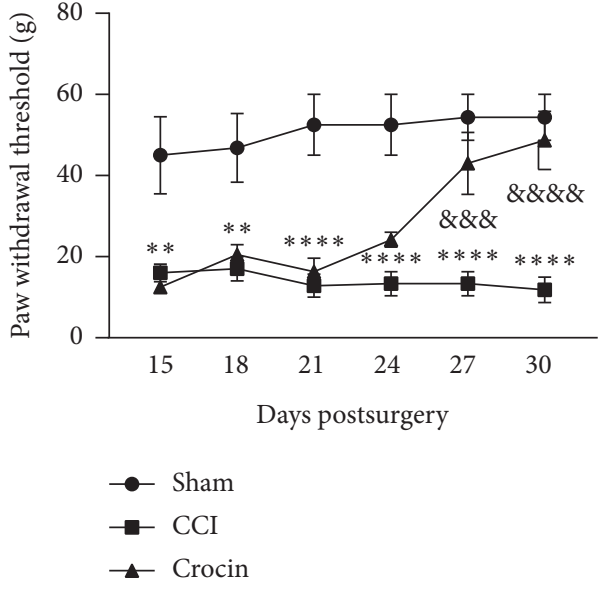

(a)

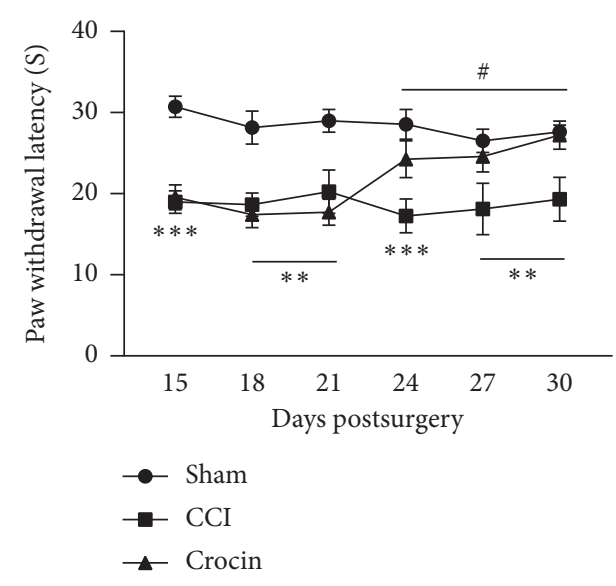

(b)

FIGURE 2: Effect of crocin on CCI-induced neuropathic pain. CCI significantly reduced mechanical (a) and thermal (b) paw withdrawal threshold compared to the sham group. Fourteen days crocin therapy significantly reduced CCI-induced mechanical allodynia and thermal hyperalgesia. Hypoalgesic effect of crocin was observed at the end of therapy (day 27) and continued on the day 30 after surgery. All data are expressed as mean \pm SEM. $n=6$. And sign and num sign compares crocin with the CCI group. Asterisks compare CCI with the sham group. $\# P<0.05,{ }^{* *} P<0.01,{ }^{* * * *} P<0.0001,{ }^{\& \& \&} P<0.01$, and ${ }^{\& \& \& \&} P<0.001$.

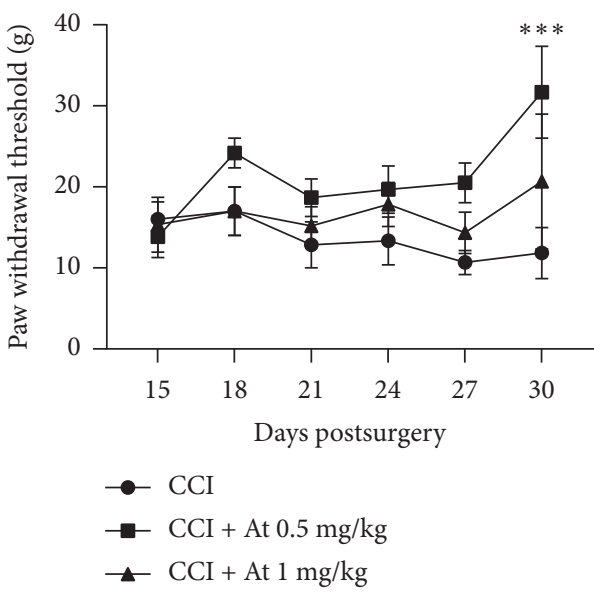

(a)

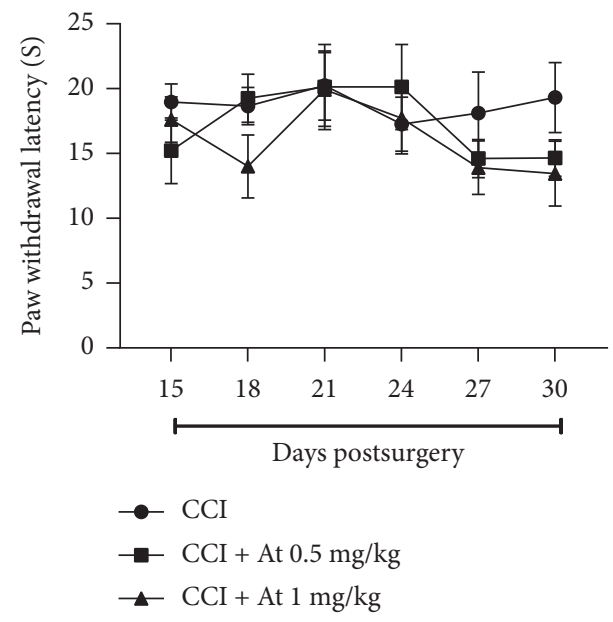

(b)

Figure 3: Effect of atropine on CCI-induced neuropathic pain. Effect of atropine with two doses, 0.5 and $1 \mathrm{mg} / \mathrm{kg}$, were examined on the mechanical allodynia (a) and thermal hyperalgesia (b) during 2 weeks of treatment from $14^{\text {th }}$ day to $27^{\text {th }}$ day after surgery. Atropine with a dose of $0.5 \mathrm{mg} / \mathrm{kg}$ significantly $(P<0.001)$ reduced mechanical allodynia at day 30 after surgery ( 3 days after stopping treatments); however, atropine $1 \mathrm{mg} / \mathrm{kg}$ did not change CCI-induced neuropathic pain during days in which pain behaviors were examined. Asterisks compare atropine $1 \mathrm{mg} / \mathrm{kg}$ with the CCI group at day 30 after surgery. All data are expressed as mean \pm SEM. $n=6$. ${ }^{* * *} P<0.001$.

$\mathrm{kg}$ (Figure 3). Depending on this result, we continued on the experiments with a dose of $1 \mathrm{mg} / \mathrm{kg}$ of atropine.

This result is in agreement with the results of other researchers that showed a dose-dependent analgesic effect for atropine [52]. They have reported that low dose $(100 \mu \mathrm{g} / \mathrm{kg})$ of atropine through presynaptic inhibition of muscarinic autoreceptors triggers acetyl choline release which in turn increases pain threshold; meanwhile, high dose of atropine $(5 \mathrm{mg} / \mathrm{kg})$ showed a hyperalgesic effect.
Therefore, in our study, it is possible that atropine with a dose of $0.5 \mathrm{mg} / \mathrm{kg}$ by triggering endogenous acetyl choline release led to increased paw withdrawal mechanical threshold. Considering the results of Ghelardini et al. [52], the administered atropine (dose of $1 \mathrm{mg} / \mathrm{kg}$ ) in the present study neither is so low dose that affects as an analgesic factor nor is high dose that affects as an hyperalgesic agent. In other words, a dose of $1 \mathrm{mg} / \mathrm{kg}$ was performed as an ineffective dose on the neuropathic pain. Furthermore, 


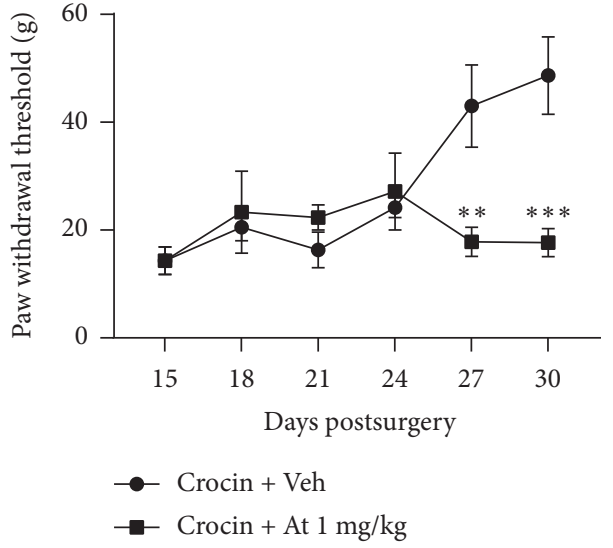

(a)

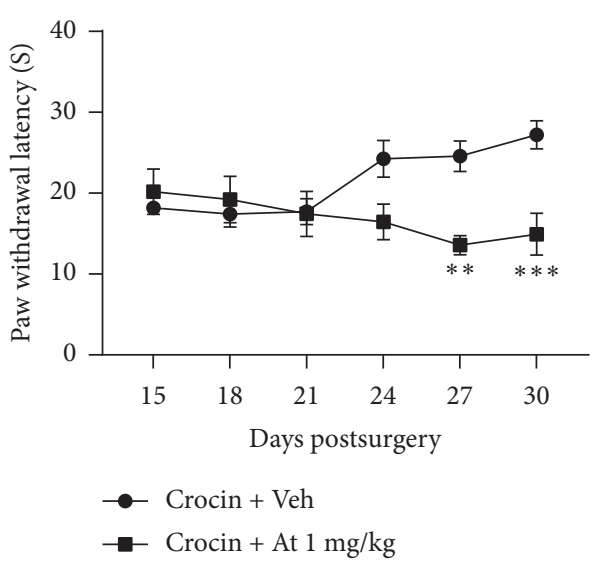

(b)

Figure 4: Effect of atropine pretreatment on the crocin-induced hypoalgesia. Atropine at $1 \mathrm{mg} / \mathrm{kg}$ significantly blocked the hypoalgesic effect of crocin. Fourteen days pretreatment with atropine significantly inhibited the effect of crocin on the mechanical allodynia (a) and thermal hyperalgesia (b), and these effects continued on to the $30^{\text {th }}$ day after surgery, while all treatments were stopped three days before. Asterisks compare atropine + crocin against that crocin group at days 27 and 30 after surgery. All data are expressed as mean \pm SEM. $n=6$. ${ }^{* *} P<0.01 ;{ }^{* * *} P<0.001$.

it has been reported that electroacupuncture analgesia was inhibited using atropine $(1 \mathrm{mg} / \mathrm{kg})$ pretreatment in male rats [53]. Moreover, activation of spinal muscarinic M2 receptors following administration of cholinesterase inhibitors lead to analgesia [54].

We found that atropine administration before crocin inhibited the hypoalgesic effect of crocin. The present finding reveals that muscarinic receptors have a role in the effects of crocin on the neuropathic pain. This finding is in agreement with the result of other researchers that showed intrathecal administration of atropine completely inhibited electroacupuncture- (modified acupuncture technique using electrical stimulation) induced antiallodynia [55], which mediated through spinal muscarinic cholinergic receptors. Furthermore, there is a report that shows saffron significantly attenuated scopolamine-induced memory impairment [28]. Therefore, this study points out that the effect of saffron mediated through the cholinergic system. It should be mentioned that several studies have revealed that the memory improving effect of saffron is related to its active constituent crocin [56, 57]. In the present study, it would be much clearer, if we had a positive control group. However, because of financial problems, we could not provide specific agonists of muscarinic receptors or more specific antagonists to compare results, and this is one of the limitations of this study. Considering the effect of crocin on the neuropathic pain following 14 days of treatment, it is possible that crocin has led to histological or even gene expression changes that in turn led to hypoalgesia, and atropine pretreatment prevented these effects. Unfortunately, due to financial problem, we could not evaluate histological changes or gene expression following crocin therapy.
However, a more detailed study can be obtained using specific agonist and antagonist or immunohistochemistry technique that results in more accurate results. We hope that we will be able to evaluate these in future research.

Regarding studies mentioned above and the present report, it seems that crocin can be suggested as a therapeutic agent against neuropathic pain, and in addition, its interaction with cholinergic antagonist's especially muscarinic must be noticed.

\section{Conclusion}

Crocin reduces CCI-induced neuropathic pain and atropine as a muscarinic antagonist blocks crocin-induced hypoalgesia. Therefore, some of hypoalgesic effects of crocin probably occur through interaction with the cholinergic system.

\section{Data Availability}

The data used to support the findings of this study are available from the corresponding author upon request.

\section{Conflicts of Interest}

The authors declare that there are no conflicts of interest.

\section{Authors' Contributions}

Abbas Ali Vafaei and Hossein Ali Safakhah carried out the design and coordinated the study. Simin Jafari, Azin Tavasoli, and Ali Ghanbari carried out all the experiments. Abbas Ali Vafaei, Hossein Ali Safakhah, and Ali Ghanbari carried out the analysis of data. Abbas Ali Vafaei, Simin 
Jafari, Azin Tavasoli, and Ali Ghanbari participated in manuscript preparation.

\section{Acknowledgments}

This project was supported by a grant (92/332111) from the Semnan University of Medical Sciences (Semnan, Iran).

\section{References}

[1] P. J. Austin and G. Moalem-Taylor, "The neuro-immune balance in neuropathic pain: involvement of inflammatory immune cells, immune-like glial cells and cytokines," Journal of Neuroimmunology, vol. 229, no. 1-2, pp. 26-50, 2010.

[2] D. Bridges, S. W. N. Thompson, and A. S. C. Rice, "Mechanisms of neuropathic pain," British Journal of Anaesthesia, vol. 87, no. 1, pp. 12-26, 2001.

[3] T. S. Jensen, R. Baron, M. Haanpää et al., "A new definition of neuropathic pain," Pain, vol. 152, no. 10, pp. 2204-2205, 2011.

[4] J. Ferrier, M. Bayet-Robert, R. Dalmann et al., "Cholinergic neurotransmission in the posterior insular cortex is altered in preclinical models of neuropathic pain: key role of muscarinic M2 receptors in donepezil-induced antinociception," Journal of Neuroscience, vol. 35, no. 50, pp. 16418-16430, 2015.

[5] A. Duttaroy, J. Gomeza, J.-W. Gan et al., "Evaluation of muscarinic agonist-induced analgesia in muscarinic acetylcholine receptor knockout mice," Molecular Pharmacology, vol. 62, no. 5, pp. 1084-1093, 2002.

[6] B. A. Clayton, K.-i. Hayashida, S. R. Childers, R. Xiao, and J. C. Eisenach, "Oral donepezil reduces hypersensitivity after nerve injury by a spinal muscarinic receptor mechanism," Anesthesiology, vol. 106, no. 5, pp. 1019-1025, 2007.

[7] M. Kimura, K.-i. Hayashida, J. C. Eisenach, S. Saito, and H. Obata, "Relief of hypersensitivity after nerve injury from systemic donepezil involves spinal cholinergic and $\gamma$-aminobutyric acid mechanisms," Anesthesiology, vol. 118, no. 1, pp. 173-180, 2013.

[8] T. C. Mendes, F. Antunes, M. M. Trachez et al., “Antihyperalgesic effects of a novel muscarinic agonist (LASSBio-873) in spinal nerve ligation in rats," Clinical and Experimental Pharmacology and Physiology, vol. 40, no. 7, pp. 404-411, 2013.

[9] C. Ghelardini, N. Galeotti, M. Calvani, L. Mosconi, R. Nicolai, and A. Bartolini, "Acetyl-l-carnitine induces muscarinic antinocieption in mice and rats," Neuropharmacology, vol. 43, no. 7, pp. 1180-1187, 2002.

[10] K. C. P. Vissers, "The clinical challenge of chronic neuropathic pain," Disability and Rehabilitation, vol. 28, no. 6, pp. 343-349, 2006.

[11] M. Gunjan and A. Ahmad, "Marketing trends and future prospects of herbal medicine in the treatment of various disease," World Journal of Pharmaceutical Research, vol. 4, no. 9, pp. 132-155, 2015.

[12] W. M. Bandaranayake, "Quality control screening toxicity and regulation of herbal drugs," in Modern Phytomedicine: Turning Medicinal Plants into Drugs, I. Ahmad, F. Aqil, and M. Owais, Eds., pp. 25-57, Wiley-VCH Verlag GmbH \& Co. KGaA, Weinheim, Germany, 2006.

[13] I. Haq, "Safety of medicinal plants," Pakistan Journal of Medical Research, vol. 43, no. 4, pp. 203-210, 2004.

[14] R. Almeida, D. S. Navarro, and J. M. Barbosa-Filho, "Plants with central analgesic activity," Phytomedicine, vol. 8, no. 4, pp. 310-322, 2001.

[15] P. A. Tarantilis, G. Tsoupras, and M. Polissiou, "Determination of saffron (Crocus sativus L.) components in crude plant extract using high-performance liquid chromatographyUV-visible photodiode-array detection-mass spectrometry," Journal of Chromatography A, vol. 699, no. 1-2, pp. 107-118, 1995.

[16] F. I. Abdullaev, "Biological effects of saffron," BioFactors (Oxford, England), vol. 4, no. 2, pp. 83-86, 1993.

[17] M. Rahimi, "Chemical and medicinal properties of saffron," Bulletin Environment Pharmacology Life Science, vol. 4, pp. 69-81, 2015.

[18] H. Hosseinzadeh and H. M. Younesi, "Antinociceptive and anti-inflammatory effects of Crocus sativus L. stigma and petal extracts in mice," BMC Pharmacology, vol. 2, no. 1, p. 7, 2002.

[19] K. Premkumar, S. K. Abraham, S. T. Santhiya, and A. Ramesh, "Protective effects of saffron (Crocus sativus Linn.) on genotoxins-induced oxidative stress in Swiss albino mice," Phytotherapy Research, vol. 17, no. 6, pp. 614-617, 2003.

[20] M. Schmidt, G. Betti, and A. Hensel, "Saffron in phytotherapy: pharmacology and clinical uses," Wiener Medizinische Wochenschrift, vol. 157, no. 13-14, pp. 315-319, 2007.

[21] M. Mazidi, M. Shemshian, S. H. Mousavi et al., "A doubleblind, randomized and placebo-controlled trial of Saffron (Crocus sativus L.) in the treatment of anxiety and depression," Journal Complementary Integrative Medicine, vol. 13, no. 2, pp. 195-199, 2016.

[22] L. Kashani, S. Esalatmanesh, F. Eftekhari et al., "Efficacy of Crocus sativus (saffron) in treatment of major depressive disorder associated with post-menopausal hot flashes: a double-blind, randomized, placebo-controlled trial," Archives of Gynecology and Obstetrics, vol. 297, no. 3, pp. 717-724, 2018.

[23] A. Ghajar, S. Neishabouri, N. Velayati et al., "Crocus sativus L. versus citalopram in the treatment of major depressive disorder with anxious distress: a double-blind, controlled clinical trial," Pharmacopsychiatry, vol. 50, no. 4, pp. 152-160, 2017.

[24] B. Ghadrdoost, A. A. Vafaei, A. Rashidy-Pour et al., "Protective effects of saffron extract and its active constituent crocin against oxidative stress and spatial learning and memory deficits induced by chronic stress in rats," European Journal of Pharmacology, vol. 667, no. 1-3, pp. 222-229, 2011.

[25] R. Srivastava, H. Ahmed, R. Dixit, Dharamveer, and S. Saraf, "Crocus sativus L.: a comprehensive review," Pharmacognosy Reviews, vol. 4, no. 8, pp. 200-208, 2010.

[26] F. Berger, A. Hensel, and K. Nieber, "Saffron extract and trans-crocetin inhibit glutamatergic synaptic transmission in rat cortical brain slices," Neuroscience, vol. 180, pp. 238-247, 2011.

[27] H. Ettehadi, S. N. Mojabi, M. Ranjbaran et al., "Aqueous extract of saffron (Crocus sativus) increases brain dopamine and glutamate concentrations in rats," Journal of Behavioral and Brain Science, vol. 3, no. 3, pp. 315-319, 2013.

[28] N. Pitsikas and N. Sakellaridis, "Crocus sativus L. extracts antagonize memory impairments in different behavioural tasks in the rat," Behavioural Brain Research, vol. 173, no. 1, pp. 112-115, 2006.

[29] G. D. Geromichalos, F. N. Lamari, M. A. Papandreou et al., "Saffron as a source of novel acetylcholinesterase inhibitors: molecular docking and in vitro enzymatic studies," Journal of Agricultural Food Chemistry, vol. 60, no. 24, pp. 6131-6138, 2012.

[30] A. Hensel, M. Niehues, M. Lechtenberg, B. Quandt, D. Schepmann, and B. Wünsch, "Analytical and functional aspects on saffron from Crocus sativus L.: development of 
quality control methods, species assortment and affinity to sigma-1 and NMDA receptors," Planta Medica, vol. 72, no. 11, p. P_074, 2006.

[31] A. M. Deslauriers, A. Afkhami-Goli, A. M. Paul et al., "Neuroinflammation and endoplasmic reticulum stress are coregulated by crocin to prevent demyelination and neurodegeneration," The Journal of Immunology, vol. 187, no. 9, pp. 4788-4799, 2011.

[32] K. N. Nam, Y.-M. Park, H.-J. Jung et al., "Anti-inflammatory effects of crocin and crocetin in rat brain microglial cells," European Journal of Pharmacology, vol. 648, no. 1-3, pp. 110-116, 2010.

[33] G. Georgiadou, V. Grivas, P. A. Tarantilis, and N. Pitsikas, "Crocins, the active constituents of Crocus Sativus L., counteracted ketamine-induced behavioural deficits in rats," Psychopharmacology, vol. 231, no. 4, pp. 717-726, 2014.

[34] E. Tamaddonfard, A.-A. Farshid, K. Eghdami, F. Samadi, and A. Erfanparast, "Comparison of the effects of crocin, safranal and diclofenac on local inflammation and inflammatory pain responses induced by carrageenan in rats," Pharmacology Reports, vol. 65, no. 5, pp. 1272-1280, 2013.

[35] M. Karami, S. Z. Bathaie, T. Tiraihi, M. Habibi-Rezaei, J. Arabkheradmand, and S. Faghihzadeh, "Crocin improved locomotor function and mechanical behavior in the rat model of contused spinal cord injury through decreasing calcitonin gene related peptide (CGRP)," Phytomedicine, vol. 21, no. 1, pp. 62-67, 2013.

[36] B. Amin and H. Hosseinzadeh, "Evaluation of aqueous and ethanolic extracts of saffron, Crocus sativus L., and its constituents, safranal and crocin in allodynia and hyperalgesia induced by chronic constriction injury model of neuropathic pain in rats," Fitoterapia, vol. 83, no. 5, pp. 888-895, 2012.

[37] E. Tamaddonfard, E. Tamaddonfard, A. A. Farshid, and A. Hamidhoseyni, "Crocin enhanced functional recovery after sciatic nerve crush injury in rats," Iran Journal of Basic Medical Science, vol. 16, no. 1, pp. 83-90, 2013.

[38] A. Vakili, M. R. Einali, and A. R. Bandegi, "Protective effect of crocin against cerebral ischemia in a dose-dependent manner in a rat model of ischemic stroke," Journal Stroke and Cerebrovascular Disease, vol. 23, no. 1, pp. 106-113, 2014.

[39] S. Amiri, Z. Jafarian, A. A. Vafaei, Z. Motaghed-Larijani, S. A. Samaei, and A. Rashidy-Pour, "Glucocorticoids interact with cholinergic system in impairing memory reconsolidation of an inhibitory avoidance task in mice," Basic Clinical Neuroscience, vol. 6, no. 3, pp. 155-162, 2015.

[40] G. J. Bennett and Y. K. Xie, "A peripheral mononeuropathy in rat that produces disorders of pain sensation like those seen in man," Pain, vol. 33, no. 1, pp. 87-107, 1988.

[41] K. Ren, "An improved method for assessing mechanical allodynia in the rat," Physiology and Behavior, vol. 67, no. 5, pp. 711-716, 1999.

[42] H. A. Safakhah, N. Moradikor, A. Bazargani et al., "Forced exercise attenuates neuropathic pain in chronic constriction injury of male rat: an investigation of oxidative stress and inflammation," Journal of Pain Research, vol. 10, pp. 14571466, 2017.

[43] H. A. Safakhah, T. Taghavi, A. Rashidy-Pour et al., "Effects of saffron (crocus sativus L.) stigma extract and its active constituent crocin on neuropathic pain responses in a rat model of chronic constriction injury," Iran Journal Pharmaceutical Research, vol. 15, no. 1, pp. 253-261, 2016.

[44] Y. S. Gwak and C. E. Hulsebosch, "Neuronal hyperexcitability: a substrate for central neuropathic pain after spinal cord injury," Current Pain and Headache Reports, vol. 15, no. 3, pp. 215-222, 2011.

[45] M. G. Liu and J. Chen, "Roles of the hippocampal formation in pain information processing," Neuroscience Bulletin, vol. 25, no. 5, pp. 237-266, 2009.

[46] T. J. Boucher, "Potent analgesic effects of GDNF in neuropathic pain states," Science, vol. 290, no. 5489, pp. 124-127, 2000.

[47] G. Negi, A. Kumar, R. P. Joshi, and S. S. Sharma, "Oxidative stress and Nrf2 in the pathophysiology of diabetic neuropathy: old perspective with a new angle," Biochem Biophys Res Commun, vol. 408, no. 1, pp. 1-5, 2011.

[48] J. N. Campbell and R. A. Meyer, "Mechanisms of neuropathic pain," Neuron, vol. 52, no. 1, pp. 77-92, 2006.

[49] X. M. Wang, T. J. Lehky, J. M. Brell, and S. G. Dorsey, "Discovering cytokines as targets for chemotherapy-induced painful peripheral neuropathy," Cytokine, vol. 59, no. 1, pp. 3-9, 2012.

[50] G. Schechtmann, Z. Song, C. Ultenius, B. A. Meyerson, and B. Linderoth, "Cholinergic mechanisms involved in the pain relieving effect of spinal cord stimulation in a model of neuropathy," Pain, vol. 139, no. 1, pp. 136-145, 2008.

[51] R. Radhakrishnan and K. A. Sluka, "Spinal muscarinic receptors are activated during low or high frequency TENSinduced antihyperalgesia in rats," Neuropharmacology, vol. 45, no. 8, pp. 1111-1119, 2003.

[52] C. Ghelardini, P. Malmberg-Aiello, A. Giotti, M. Malcangio, and A. Bartolini, "Investigation into atropine-induced antinociception," British Journal of Pharmacology, vol. 101, no. 1, pp. 49-54, 1990.

[53] Y. H. Baek, D. Y. Choi, H. I. Yang, and D. S. Park, “Analgesic effect of electroacupuncture on inflammatory pain in the rat model of collagen-induced arthritis: mediation by cholinergic and serotonergic receptors," Brain Research, vol. 1057, no. 1-2, pp. 181-185, 2005.

[54] H. L. Pan, Z.-Z. Wu, H. Y. Zhou, S.-R. Chen, H.-M. Zhang, and D.-P. Li, "Modulation of pain transmission by G-proteincoupled receptors," Pharmacology Therapeutics, vol. 117, no. 1, pp. 141-161, 2008.

[55] J. H. Park, S. K. Kim, H. N. Kim et al., "Spinal cholinergic mechanism of the relieving effects of electroacupuncture on cold and warm allodynia in a rat model of neuropathic pain," Journal of Physiology Science, vol. 59, no. 4, pp. 291-298, 2009.

[56] H. Hosseinzadeh, H. R. Sadeghnia, F. A. Ghaeni, V. S. Motamedshariaty, and S. A. Mohajeri, "Effects of saffron (Crocus sativus L.) and its active constituent, crocin, on recognition and spatial memory after chronic cerebral hypoperfusion in rats," Phytotheraphy Research, vol. 26, no. 3, pp. 381-386, 2012.

[57] K. Abe and H. Saito, "Effects of saffron extract and its constituent crocin on learning behaviour and long-term potentiation," Phytotheraphy Research, vol. 14, no. 3, pp. 149-152, 2000. 